\title{
Sazonalidade e dieta frugívora do saí-andorinha Tersina viridis (Illiger, 1911) em reflorestamento da mata ciliar do Rio Mogi Guaçu, São Paulo, Brasil
}

\author{
Paulo Rubim ${ }^{1,2}$ \\ ${ }^{1}$ Grupo de Fenologia e Dispersão de Sementes, Laboratório de Biologia da Conservação, \\ Departamento de Ecologia, Universidade Estadual Paulista - UNIP, \\ CP 199, 13506-900 Rio Claro, SP, Brasil. \\ 2Auemail: paulo.rubim@yahoo.com.br
}

RUBIM, P. Seasonality and frugivory by swallow-tanager Tersina viridis (Illiger, 1911) in reforested gallery forest of the Mogi Guaçu river, São Paulo, Brazil. Biota Neotrop., 9(3): http://www.biotaneotropica.org.br/ v9n3/en/abstract?article+bn01709032009.

Abstract: According to the literature, Tersina viridis (Illiger, 1911) has seasonal habit concerning to its local displacements. However, the reasons of such displacements are still poorly known. In reforested gallery forest area of the Mogi-Guaçu river, São Paulo state, Brazil, i quantified the annual variation of the population density of T. viridis throughout a year period every month. The number of individuals of the species increased substantially during the dry season, whereas in the rainy season no individual was observed. These findings suggest a seasonal behavior of the species in this site. They also suggest that $T$. viridis probably has different routes of displacement in large flocks during the dry season in the interior of the São Paulo state and surroundings. The larger abundances of some ornithocoric plant species may explain the routes of such displacements in the studied site, rather than the general pattern of fruiting of the ornithocoric species.

Keywords: seasonality, ornitocoric plant, Tersina viridis, Trema micrantha.

RUBIM, P. Sazonalidade e dieta frugívora do saí-andorinha Tersina viridis (Illiger, 1911) em reflorestamento da mata ciliar do Rio Mogi Guaçu, São Paulo, Brasil. Biota Neotrop., 9(3): http://www.biotaneotropica.org. br/v9n3/pt/abstract?article+bn01709032009.

Resumo: Segundo a literatura, Tersina viridis (Illiger, 1911) possui hábito gregário sazonal em deslocamentos locais. Os reais motivos desses deslocamentos ainda são poucos conhecidos. Em reflorestamento de mata ciliar do rio Mogi-Guaçu, SP, foi possível quantificar a variação sazonal mensal na densidade populacional durante um ano. O número de indivíduos aumentou substancialmente durante a estação seca e ausência durante a estação chuvosa, assim, caracterizando um comportamento fortemente sazonal de ocorrência nesse local. Provavelmente T. viridis possui algumas rotas de deslocamentos quando aparecem em grandes agregados no período mais seco na região do interior do estado de São Paulo e vizinhanças. A grande abundância de algumas espécies de plantas ornitocóricas pode ser alguns dos motivos que determinam a rota desses deslocamentos na região estudada, e não o padrão geral da fenologia da frutificação das espécies ornitocóricas.

Palavras-chave: sazonalidade, plantas ornicocóricas, Tersina viridis, Trema micrantha. 


\section{Introdução}

Devido ao comportamento de deslocamentos locais sazonais em toda sua área de distribuição no neotrópico, Tersina viridis (Illiger, 1911) (Emberezidae-Thraupinae), popularmente conhecida como saíandorinha é considerada uma espécie de ave hábitos gregários e semimigratória (Schaefer 1953, Hayes et al. 1994, Sick 1997, Hilty 2003). Aparentemente, as populações de $T$. viridis que ocorrem na região do estado de São Paulo, sul de Minas Gerais e norte do Paraná possuem deslocamentos sazonais influenciados pela sazonalidade climática da região e pela frutificação de algumas espécies ornitocóricas. Nessa região, estudos de no mínimo um ano de acompanhamento mensal da avifauna relatam os primeiros registros de chegada de T. viridis ao final da estação chuvosa, entre os meses de fevereiro e março, e o desaparecimento entre o final da estação seca e início da chuvosa, entre os meses de junho a setembro no interior do estado de São Paulo (Monteiro et al. 1992, Aleixo \& Viellieard 1995, Lima \& Aleixo 2000, Willis \& Oniki 2003, Pozza \& Pires 2003), sul de Minas Gerais (Franchin \& Marçal-Junior 2002) e norte do Paraná (Lopes \& Anjos 2006).

Em geral, T. viridis também possui variações sazonais ao longo do ano em relação à sua dieta, quando pode apresentar tendências à insetivoria durante o verão e frugivoria no inverno (Schaefer 1953, Sick 1997, Manhães 2003). No sudeste brasileiro, vários estudos evidenciaram essa relação sazonal de $T$. viridis com plantas hemiparasitas (erva-de-passarinho) da família Loranthaceae e a exótica Michelia champaca L. (Meliaceae), nos quais os autores enfatizam a habilidade de $T$. viridis em fazer deslocamentos locais em busca dessas fruteiras (Monteiro et al. 1992, Lombardi \& Motta-Junior 1993, Figueiredo 1997, Sick 1997, Guerra \& Marini 2002, Cazetta \& Galetti 2003).

Em regiões tropicais muitas espécies frugívoras realizam deslocamentos migratórios por diversos motivos, como a sazonalidade climática (Loiselle 1987, Blake et al. 1992), mudança estrutural no hábitat (Borghesio \& Laiolo 2004a) e principalmente em relação à sazonalidade na abundância de frutos (Greenberg 1981, Levey 1988, Blake \& Loiselle 1991, Ganesh \& Davidar 1999, Hanya 2005). Até o momento nenhum trabalho quantificou a abundância sazonal de ocorrência de uma população em deslocamento de $T$. viridis e sua relação com árvores frutíferas. Dessa forma, nesse trabalho descrevo a densidade sazonal e registros de sua alimentação frugívora durante um ano de estudo em reflorestamento de mata ciliar na região do médio rio Mogi Guaçu, sudeste do Brasil.

\section{Material e Métodos}

Conduzi esse estudo na Reserva Particular do Patrimônio Natural (RPPN) São Marcelo (22 21' 58"' S e 46 $58^{\circ}$ '22”' O), localizada à margem esquerda do rio Mogi Guaçu, Mogi Guaçu, estado de São Paulo. Sua vegetação é constituída por dois reflorestamentos de espécies nativas - um com cerca de 30 anos e outro com um ano de idade, totalizando cerca de 187,03 ha nos dois reflorestamentos. No reflorestamento de 30 anos Trema micrantha (L.) Blume, Guazuma ulmifolia Lam. e Schizolobium parahyba (Vell.) S.F. Blake são as espécies mais abundantes. O dossel raramente ultrapassa oito metros de altura em espaçamento de $2 \times 3 \mathrm{~m}$, e sub-bosque praticamente sem recrutamento devido à invasão de capim-braquiária - Brachiaria decumbens Stapf. O reflorestamento de um ano (plantado em 2002) possui 100 espécies nativas plantadas em espaçamento de $2 \times 3 \mathrm{~m}$. Devido a pouca idade deste reflorestamento durante o período de estudo as árvores possuíam tamanhos inferiores a $2 \mathrm{~m}$ de altura e somente Schizolobium parahyba ultrapassava essa medida.

Entre o período de 1971 a 2003, a região apresentou precipitação média anual de $1365 \mathrm{~mm}$ e temperatura média anual de $20,6{ }^{\circ} \mathrm{C}$.
O clima para a região de Mogi Guaçu é bastante sazonal, com uma estação chuvosa e quente (verão) de outubro a abril e precipitação superior a $100 \mathrm{~mm}$, e outra estação seca e fria (inverno) entre os meses de maio a setembro, onde a precipitação geralmente é inferior a $60 \mathrm{~mm}$ e déficit hídrico entre os meses de julho e agosto. Durante o período de estudo, a precipitação foi de $1411 \mathrm{~mm}$ e a temperatura média de $20,5{ }^{\circ} \mathrm{C}$. Todos os dados climáticos foram cedidos pela estação meteorológica da Estação Ecológica Mogi Guaçu localizada a cerca de $20 \mathrm{~km}$ da área estudada.

Realizei este trabalho em visitas mensais durante o período de doze meses entre março de 2003 a fevereiro de 2004. Em cada mês realizei quatro dias consecutivos de censo de densidade num transecto de $1,5 \mathrm{~km}$ retilíneo no reflorestamento de 30 anos, onde instalei 15 pontos de escuta de aves distando $100 \mathrm{~m}$ entre os mesmos. Utilizei cinco minutos de permanência em cada um dos pontos entre 6:00 hàs 10:00 h da manhã, sendo que o início foi dado depois de um minuto após a chegada a cada ponto para não afugentar as aves enquanto caminhava até o mesmo. $\mathrm{O}$ censo de aves foi baseado em método proposto por Reynolds et al.(1980), onde registrei todos os indivíduos de $T$. viridis encontrados dentro de um raio de observação partir do centro de cada ponto. Utilizei $40 \mathrm{~m}$ de raio máximo de inclusão devido à dificuldade de visualização dos indivíduos de $T$. viridis além dessa distância e não considerei indivíduos em vôo. Dessa forma, para calculo da densidade mensal utilizei o somatório dos registros dos 15 pontos e quatro dias, dividindo-se somatório da área em que a ave teve presente (para maiores detalhes ver Reynolds et al.(1980)). Ainda nesse transecto realizei duas excursões mensais (ca. $1 \mathrm{~km} / \mathrm{h}$ ), entre às 16:00 e 18:00 h onde coletei o número de registros de alimentação ("feeding bouts") de T. viridis com as plantas ornitocóricas, conforme proposto por Galetti et al. (2003). Quantifiquei o padrão fenológico da frutificação através de caminhadas não padronizadas durante os quatro dias de pesquisas na RPPN. Esse método proposto por Batalha \& Mantovani (2000) considerada uma espécie em frutificação mesmo quando somente um indivíduo da população apresentou frutos.

\section{Resultados}

A população de Tersina viridis apresentou alta sazonalidade de ocorrência no reflorestamento durante o ano, com significativo número de indivíduos entre março a julho (Figura 1). Durante o censo de registros de alimentação foi possível observar um indivíduo em janeiro e um em fevereiro.

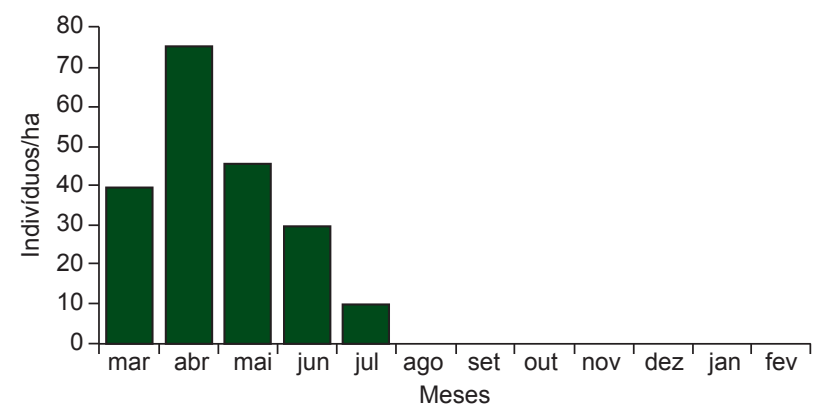

Figura 1. Densidade de Tersina viridis durante o período de estudo (março de 2003 a fevereiro de 2004) na RPPN Parque São Marcelo, Mogi Guaçu, São Paulo, Brasil.

Figure 1. Tersina viridis density during the study period (March of 2003 to February of 2004) at RPPN São Marcelo, Mogi Guaçu, São Paulo, Brazil. 
Tabela 1. Fenologia da frutificação de plantas ornitocóricas na RPPN Parque São Marcelo durante o período de estudo (março de 2003 a fevereiro de 2004), Mogi Guaçu, São Paulo, Brasil. Números em Trema micrantha e Nectandra megapotamica $=$ "feeding bout"/km percorrido. Cinza (agosto a dezembro) $=$ ausência de Tersina viridis na área de estudo, * presença de frutos.

Table 1. Frutification phenology from ornitocoric's plants at RPPN Parque São Marcelo during the study period (march of 2003 to february of 2004), Mogi Guaçu, São Paulo, Brazil. Numbers showed in Trema micrantha and Nectandra megapotamica $=$ feeding bout $/ \mathrm{km}$. Gray (august to december) $=$ Tersina viridis absent in the study area, * with fruit.

\begin{tabular}{|c|c|c|c|c|c|c|c|c|c|c|c|c|}
\hline \multirow[t]{2}{*}{ Espécie } & \multicolumn{10}{|c|}{2003} & \multicolumn{2}{|c|}{2004} \\
\hline & mar & abr & mai & jun & jul & ago & set & out & nov & dez & jan & fev \\
\hline \multicolumn{13}{|l|}{ CECROPIACEAE } \\
\hline Cecropia pachystachya Trécul & $*$ & $*$ & $*$ & $*$ & $*$ & - & - & $*$ & $*$ & - & $*$ & - \\
\hline \multicolumn{13}{|l|}{ ULMACEAE } \\
\hline Trema micrantha (L.) Blume & 1,5 & 19 & 5 & 5,5 & $*$ & $*$ & $*$ & - & - & $*$ & $*$ & 1 \\
\hline \multicolumn{13}{|l|}{ ANACARDIACEAE } \\
\hline Schinus terebinthifolius Raddi & $*$ & * & $*$ & $*$ & $*$ & - & - & - & - & - & $*$ & $*$ \\
\hline \multicolumn{13}{|l|}{ RHAMNACEAE } \\
\hline Hovenia dulcis Thunb. & $*$ & - & - & - & - & - & - & - & - & - & - & - \\
\hline \multicolumn{13}{|l|}{ MELIACEAE } \\
\hline Melia azedarach $\mathrm{L}$. & - & $*$ & * & $*$ & $*$ & - & - & - & - & - & - & - \\
\hline \multicolumn{13}{|l|}{ VISCACEAE } \\
\hline Phoradendron sp. & - & $*$ & - & $*$ & - & - & - & - & - & - & - & - \\
\hline \multicolumn{13}{|l|}{ VITACEAE } \\
\hline Cissus sp. & - & * & - & $*$ & - & - & - & - & - & - & - & - \\
\hline \multicolumn{13}{|l|}{ RUTACEAE } \\
\hline Zanthoxyllum sp. & - & - & $*$ & - & - & - & $*$ & - & - & - & - & - \\
\hline \multicolumn{13}{|l|}{ FABACEAE } \\
\hline Copaifera langsdorffii Desf. & - & - & - & $*$ & - & - & - & - & - & - & - & - \\
\hline \multicolumn{13}{|l|}{ ARALIACEAE } \\
\hline Schefflera sp. & - & - & - & - & $*$ & $*$ & $*$ & - & - & - & - & - \\
\hline \multicolumn{13}{|l|}{ SOLANACEAE } \\
\hline Solanum americanum Mill. & - & - & - & - & $*$ & $*$ & $*$ & - & - & - & - & - \\
\hline Cestrum sendtenerianum Mart. ex Sendt & - & - & - & - & - & - & $*$ & - & - & - & - & - \\
\hline Solanum agrarium Sendtn. & - & - & - & - & - & - & $*$ & - & - & - & - & - \\
\hline \multicolumn{13}{|l|}{ MORACEAE } \\
\hline Morus nigra $\mathrm{L}$. & - & - & - & - & - & - & - & $*$ & $*$ & - & - & - \\
\hline \multicolumn{13}{|l|}{ LAURACEAE } \\
\hline Nectandra megapotamica (Spreng.) Mez & - & - & - & - & - & - & - & - & - & - & 5 & $*$ \\
\hline \multicolumn{13}{|l|}{ MYRTACEAE } \\
\hline Psidium guajava $\mathrm{L}$. & - & - & - & - & - & - & - & - & - & - & $*$ & $*$ \\
\hline \multicolumn{13}{|l|}{ RAMNACEAE } \\
\hline Ramnidium elaeocarpus Reissek. & - & - & - & - & - & - & - & - & - & - & $*$ & $*$ \\
\hline \multicolumn{13}{|l|}{ BORAGINACEAE } \\
\hline Cordia trichotoma (Vell.) Arráb. ex Steud. & - & - & - & - & - & - & - & - & - & - & - & $*$ \\
\hline \multicolumn{13}{|l|}{ VERBENACEAE } \\
\hline Cytharexyllum myrianthum Cham. & - & - & - & - & - & - & - & - & - & - & - & $*$ \\
\hline Total de espécies frutificando por mês & 4 & 6 & 5 & 6 & 7 & 2 & 6 & 2 & 2 & 1 & 6 & 7 \\
\hline
\end{tabular}

Através do estudo da fenologia da frutificação foi possível observar 19 espécies de plantas ornitocóricas frutificando durante o período de estudo, onde julho e fevereiro foram os meses com maior número de espécies (7) e novembro o menor (1) (Tabela 1). Cecropia pachystachya, Trema micrantha e Schinus terebinthifolius Raddi foram as espécies que frutificaram durante o maior número de meses no ano de estudo.
Os registros de alimentação de $T$. viridis ocorreram de março a julho e fevereiro se alimentando de Trema micrantha (Ulmaceae) e no mês de janeiro se alimentando de Nectandra megapotamica (Spreng.) Mez (Lauraceae). No mês de abril foi possível registrar 19 indivíduos $/ \mathrm{km}$ se alimentando de $T$. micrantha (Tabela 1). Dessa forma, se somarmos os números de meses com frutos disponíveis coincidentes com o intervalo de permanência de Tersina na área, 
percebemos que somente $14 \%$ da proporção de meses com frutos disponíveis foram utilizados durante o ano.

\section{Discussão}

A grande abundância de $T$. viridis ocorreu em apenas alguns meses da estação seca, evidenciando forte sazonalidade da população amostrada. A maior densidade de espécies de aves frugívoras durante o período mais seco do ano também ocorre em outras regiões da América Central (Greenberg 1981) e África (Borghesio \& Laiolo 2004b), onde essas aves agregam-se em bandos durante o período mais seco do ano a procura de frutos durante o forrageio. A alta sazonalidade de T. viridis na área de estudo, entre março a junho, é similar temporalmente a outros registros no interior do estado de São Paulo (Monteiro et al. 1992, Aleixo \& Vielliard 1995, Lima \& Aleixo 2000, Pozza \& Pires 2003, Willis \& Oniki 2003), norte do Paraná (Lopes \& Anjos 2006) e sul de Minas Gerais (Franchin \& Marçal-Junior 2002), onde T. viridis foi registrada durante a estação mais seca do ano.

Todos os indivíduos da população de $T$. viridis passaram pela RPPN São Marcelo sempre no sentido de orientação, Oeste para Leste, evidenciando o movimento sazonal descrito para a espécie, porém, de maneira unidirecional em escala local. As causas que motivam seus deslocamentos ainda são pouco conhecidas, sendo que alguns autores sugerem que ocorram devido à fenologia da frutificação de algumas espécies de plantas, como as ervas-de-passarinho (Monteiro et al. 1992) e Michelia champaca (Lombardi \& Motta-Junior 1993, Figueiredo 1997, Sick 1997). Muitas espécies de aves frugívoras possuem deslocamento temporal e espacial em busca de frutos (Levey 1988, Blake \& Loiselle 1991).

No presente estudo $T$. viridis apresentou forte tendência alimentar à Trema micrantha, espécie muito utilizada em reflorestamentos em São Paulo (Macedo 1993), onde preferencialmente se alimentou desta espécie apesar de outras espécies ornitocóricas frutificarem durante o mesmo período. Essa relação provavelmente se deve à alta abundância de T. micrantha frutificando ao mesmo tempo e local e que conseqüentemente, atrai grande quantidade de aves frugívoras que podem eventualmente dispersar suas sementes. Além disso, muitas espécies de aves migratórias são mais abundantes em hábitats secundários do que em florestas primárias (Waide et al. 1980, Martin 1985, Levey 1988). T. viridis pode ser uma destas espécies que freqüentam preferencialmente habitats secundários considerando que não ocorreu em fragmentos de florestas com cerca de 200 ha em outros levantamentos de espécies de aves frugívoras no interior do estado de São Paulo realizados por Galetti \& Pizo (1996), e Willis \& Oniki (2002).

Provavelmente $T$. viridis possui algumas rotas de deslocamentos quando aparecem em grandes agregados no período mais seco na região do interior do estado de São Paulo e vizinhanças. A grande abundância de algumas espécies de plantas ornitocóricas pode ser alguns dos motivos que determinam a rota desses deslocamentos na região estudada, e não o padrão geral da fenologia da frutificação das espécies ornitocóricas.

\section{Agradecimentos}

Agradeço a International Paper do Brasil pela ajuda logística e liberação para trabalhar na RPPN Parque São Marcelo.

\section{Referências Bibilográficas}

ALEIXO, A. \& VIELLIARD, J.M.E. 1995. Composição e dinâmica da avifauna da mata de Santa Genebra, Campinas, São Paulo, Brasil. Rev. Bras. Zool. 12(3):493-511.
BATALHA, M.A. \& MANTOVANI, W. 2000. Reproductive phenological patterns of cerrado plant species at the Pé-de-Gigante Reserve (Santa Rita do Passa Quatro, SP, Brazil): a comparison between the herbaceous and woody floras. Rev. Bras. Biol. 60(1):129-145.

BORGHESIO, L. \& LAIOLO, P. 2004a. Seasonal foraging ecology in a forest avifauna of northern Kenya. J. Trop. Ecol. 20(2):145-155.

BORGHESIO, L. \& LAIOLO, P. 2004b. Habitat use and feeding ecology of Kulal White-eye Zosterops kulalensis. Bird Conser. Int. 14(1):11-24.

BLAKE, J.G. \& LOISELLE, B.A. 1991. Variation in resource abundance affects capture rates of birds in three lowland habitats in Costa Rica. Auk. 108(1):114-130.

BLAKE, J.G., NIEMI, G.J. \& HANOWSKI, J.M. 1992. Drought and annual variation in bird populations: effects of migratory strategy and breeding habitat. In Ecology and conservation of neotropical migrant landbirds. (J.M. Hagan \& D.W. Johnston, eds.). Smithsonian Institution Press, Washington, p. 419-429.

CAZETTA, E. \& GALETTI, M. 2003. Ecologia da Erva-de-passarinho. Cienc. Hoje. 33(194):72-74.

FIGUEIREDO, R.A. 1997. Testing a biological model of adaptation for the exotic tree Michelia champaca L. (Magnoliaceae) in Brazil. Cien. Cul. 49(4):278-280.

FRANCHIN, A.G. \& MARÇAL Jr., O. 2002. A riqueza da avifauna urbana em praças de Uberlândia (MG). Horiz. Cient. 1(1):http://www.propp.ufu. br/revistaeletronica/ (último acesso em 03/07/2008).

GALETTI, M. \& PIZO, M.A. 1996. Fruit eating birds in a forest fragment in southeastern Brazil. Rev. Bras. Ornit. 4(2):71-79.

GALETTI, M., PIZO, M.A. \& MORELLATO, L.P. 2003. Fenologia, frugivoria e dispersão de sementes. In Métodos de estudos em biologia da conservação e manejo da vida silvestre (L. CÜLLEN Jr., R. RUDRAN \& C. VALLADARES-PÁDUA, eds). UFPR, Curitiba, p. 395-422.

GANESH, T. \& DAVIDAR, P. 1999. Fruit biomass and relative abundance of frugivores in a rain forest of southern Western Ghats, India. J. Trop. Ecol. 15(4):339-413.

GREENBERG, R. 1981. Frugivory in some migrant tropical forest wood warblers. Biotropica. 3(3):215-223.

GUERRA, T.J.A. \& MARINI, M.A. 2002. Bird frugivory in Struthanthus concinnus (Loranthaceae) in Southeastern Brazil. Rev. Bras. Ornit. 10(2):133-138.

HANYA, G. 2005. Comparisons of dispersal success between the species fruiting prior to and those at the peak of migrant frugivore abundance. Plant. Ecol. 181(2):167-177.

HAYES, F.E., SCHARF, P.A. \& RIDGELY, R.S. 1994. Austral bird migrants in Paraguay. The Condor. 96(1):83-97.

HILTY, S.L. 2003 Birds of Venezuela. 2 ed. Princenton University Press, EUA, p. 928.

LEVEY, D.J. 1988. Spatial and temporal variation in Costa Rican fruit and fruit-eating bird abundance. Ecol. Monogr. 58(4)251-269.

LIMA, F.C.T. \& ALEIXO, A. 2000. Notas sobre algumas aves em ambientes antropizados da cidade de Campinas, São Paulo, Brasil. Boletim CEO. $14: 2-6$.

LOISELLE, B.A. 1987. Migrant abundance in a Costa Rican lowland forest canopy. J. Trop. Ecol. 3(2)163-168.

LOMBARDI, J.A. \& MOTTA Jr., J.C. 1993. Seeds of the champak, Michelia champaca L. (Magnoliaceae) as a food source for Brazilian birds. Cien. Cul. 45(6):408-409.

LOPES, E.V. \& ANJOS, L. 2006. A composição da avifauna da Universidade Estadual de Londrina, norte do Paraná, Brasil. Rev. Bras. Zool. 23(1):145-156.

MACEDO, A.C. 1993. Revegetação: matas ciliares e de proteção ambiental. Governo do Estado de São Paulo/Fundação Florestal, São Paulo. http:// www.fflorestal.sp.gov.br/publicacao/manual_vegetacao_1ed_1993.pdf (último acesso em 03/06/2008). 
MANHÃES, M.A. 2003. Dieta de Traupíneos (Passeriformes, Emberizidae) no Parque Estadual do Ibitipoca, Minas Gerais, Brasil. Iheringia Sér. Zool. 93(1):59-73.

MARTIN, T.E. 1985. Selection of second-growth woodland by frugivorous migrating birds in Panama: an effect of fruit size and plant density? J. Trop. Ecol. 1(2):157-170.

MONTEIRO, R.F., MARTINS, R.P. \& YAMAMOTO, K. 1992. Host specificity and seed dispersal of Psittacanthus robustus (Loranthaceae) in South-Eastern Brazil. J. Trop. Ecol. 8(3):307-314.

POZZA, D.D. \& PIRES, J.S.R. 2003. Bird communities in two fragments of semideciduous forest in rural São Paulo State. Braz. J. Biol. 63(2):307-319.

REYNOLDS, R.T., SCOTT, J.M. \& NUSSBAUM , R.A. 1980. A variable circular-plot method for bird number. The Condor. 82(3):309-313.

SCHAEFER, E. 1953. Contribution to the life history of the Swallow-Tanager. Auk. 70(4):403-470.
SICK, H. 1997. Ornitologia Brasileira. Nova Fronteira, Rio de Janeiro, p.912.

WAIDE, R.B., EMLEN, J.T. \& TRAMER, E.J. 1980. Distribution of migrant birds in the Yucatan Peninsula: a survey. In Migrant birds in the neotropics: ecology, behavior, distribution, and conservation. (A. Keaste \& E.S. Morton, eds). Smithsonian Institution Press, Washington, p. $165-171$

WILLIS, E.O. \& ONIKI, Y. 2002. Birds of a central São Paulo woodlot: 1 censuses 1982-2000. Braz. J. Biol. 62(2):197-210.

WILLIS, E.O. \& ONIKI, Y. 2003. Birds of a eucaliptos woodlot in interior São Paulo. Braz. J. Biol. 63(1):141-158.

Recebido em 19/09/08 Versão Reformulada recebida em 11/06/09 Publicado em 27/07/09 